\title{
Desarrollo y validación psicométrica de un nuevo cuestionario de screening para la disfunción eréctil (Cuestionario SQUED)
}

\author{
Martín-Morales $\mathrm{A}^{1}$, Meijide Rico $\mathrm{F}^{2}$, García González $\mathrm{JI}^{3}$, Manero Font $\mathrm{M}^{4}$, García-Losa $\mathrm{M}^{5}$, \\ Artés Ferragud $\mathrm{M}^{6}$, en representación de los investigadores del proyecto ESTIMA ${ }^{7}$. \\ ${ }^{1}$ Servicio de Urología. Hospital Carlos Haya. Málaga. ${ }^{2}$ Clínica Povisa. Vigo. ${ }^{3}$ Hospital de Móstoles. Madrid. \\ ${ }^{4}$ Q.F. Bayer. Barcelona. ${ }^{5}$ GlaxoSmithKline. Madrid. ${ }^{6}$ Adelphi Targis. Barcelona. ${ }^{7}$ Ver listado de investigadores.
}

Actas Urol Esp. 2007;31(2):106-112

\section{RESUMEN}

DESARROLLO Y VALIDACIÓN PSICOMÉTRICA DE UN NUEVO CUESTIONARIO DE SCREENING PARA LA DISFUNCIÓN ERÉCTIL (CUESTIONARIO SQUED)

Introducción. Es necesario contar con herramientas sencillas que permitan detectar de forma válida y fiable la presencia de disfunción eréctil (DE). El objetivo de este estudio fue desarrollar y validar un cuestionario de cribado de la DE que fuera útil para usar en atención primaria (AP).

Material y Métodos. El desarrollo del cuestionario SQUED (Short Questionnaire for Erectile Dysfunction) incluyó la identificación de conceptos y generación de ítems, evaluación del contenido y la validez aparente a partir de entrevistas con sujetos para valorar la compresión y adecuación del lenguaje. Para la validación psicométrica se realizó un estudio epidemiológico, observacional, comparativo y multicéntrico. Los participantes completaron el cuestionario en el ámbito de la AP y fueron derivados al especialista para la confirmación diagnóstica mediante entrevista clínica y la administración del International Index of Erectile Function (IIEF). Se evaluó la consistencia interna, la fiabilidad del test-retest y la sensibilidad y la especificidad del SQUED.

Resultados. Participaron 405 sujetos, de los cuales 316 (208 con DE y 108 sin DE) fueron evaluables. El cuestionario SQUED mostró una alta consistencia interna (alfa de Cronbach $=0,92$ ) y una buena fiabilidad test-retest (indice Kappa= 0,77). Además, el cuestionario mostró una alta capacidad diagnóstica con valores de sensibilidad y especificidad, 0,87 y 0,78, respectivamente, respecto al diagnóstico del urólogo. El punto de corte se estableció en una puntuación de 12.

Discusión. El cuestionario SQUED es un instrumento sencillo, fácil de usar y fiable. Constituye una herramienta útil, y la más corta validada, para que los médicos de AP puedan identificar fácilmente pacientes con DE. Su sencillez debería hacer fácil el proceso de adaptación cultural y validación en otros idiomas.

Palabras Clave: Disfunción eréctil. Cuestionario SQUED. Validación. Atención Primaria.

\section{ABSTRACT}

DEVELOPMENT AND PSYCHOMETRIC VALIDATION OF A NEW SCREENING QUESTIONNAIRE FOR ERECTILE DYSFUNCTION (SQUED QUESTIONNAIRE)

Background. It is necessary to have simple tools to screen erectile dysfunction (ED) in an easy, reliable and valid manner. The objective of this study was to develop and validate a short diagnostic questionnaire for erectile dysfunction (SQUED), easy to use in the primary care setting.

Subjects and method. The development of SQUED included: concepts identification, item generation and evaluation of contents and face validity through interviews with subjects to assess comprehension and idiomatic adequacy. The psychometric validation was conducted in an epidemiologic, observational, comparative and multicenter study. Participants should complete the questionnaire in primary care setting and send to the specialist to confirm the diagnosis by an in depth interview and the application of IIEF questionnaire. Internal consistency, test-retest reliability and sensitivity and specificity of SQUED was evaluated.

Results. Out of 405 subjects enrolled, 316 (208 ED and 108 non-ED) were evaluable. The SQUED questionnaire showed a high internal consistency (Cronbach's alpha $=0.92$ ) and a good test-retest reliability (Kappa index $=0.77$ ). Furthermore, the questionnaire showed a good diagnostic capacity with high values of sensitivity and specificity, 0.87 and 0.78 , respectively, in relation to the diagnosis made by urologists. The cutting point was established at a score of 12 .

Discussion. The SQUED questionnaire is a simple, easy to use and reliable instrument. It can become a useful tool, and the shortest validated, for primary care physicians to easily screen for ED patients. Its simplicity should facilitate an easy cultural adaptation and validation into other languages.

Keywords: Erectile dysfunction. SQUED questionnaire. Validation. Primary care setting. 
$\mathrm{L}^{2}$ a disfunción eréctil (DE) se define como la incapacidad persistente o recurrente para conseguir o mantener la suficiente rigidez del pene que permita una relación sexual satisfactoria $^{1}$. Diversos estudios recientes han valorado la prevalencia de $\mathrm{DE}$, mostrando diferentes cifras (10\% - 52\%) dependiendo de la definición de DE y del diseño del estudio. Sin embargo, todos los estudios mostraron un aumento de la DE con la edad, en particular en hombres de 40 a 70 años $^{2,3}$.

Los resultados del primer estudio epidemiológico sobre $\mathrm{DE}^{4}$ realizado en España muestran una prevalencia del 12,1\%, implicando que aproximadamente de 1,5 a 2 millones de hombres españoles de 25 a 70 años, sufren este problema.

El reciente desarrollo de fármacos orales con un buen perfil de eficacia y seguridad ${ }^{5-7}$ para el tratamiento de la DE ha transformado su abordaje diagnóstico y terapéutico.

El desarrollo de este cuestionario de detección o cribado para la DE surge de la necesidad de contar con herramientas que permitan detectar de forma sencilla los pacientes susceptibles de padecer DE en un ámbito no especializado como son los centros de atención primaria (AP). Aunque ya existen intentos previos de desarrollar un instrumento simplificado ${ }^{8}$ partiendo del IIEF $^{9}$, en nuestra opinión, los 5 items resultantes son todavía demasiado complejos para ser utilizados cómoda y eficientemente por profesionales de salud no especializados en esta área. El objetivo del presente estudio fue diseñar $\mathrm{y}$ validar un cuestionario breve de detección o diagnóstico inicial de la DE.

\section{MATERIAL Y MÉTODOS \\ Diseño del estudio y población}

Se llevó a cabo un estudio epidemiológico, transversal, observacional, comparativo y multicéntrico con el objetivo de validar un cuestionario diagnóstico breve para la DE en español (SQUED - short diagnostic questionnaire for ED) en AP. Los sujetos incluidos en el estudio, a través de los médicos de AP, fueron hombres mayores de 18 años, con sospecha de DE por los síntomas que presentaban o que estaban recogidos en su historia clínica. Los participantes debían completar el cuestionario SQUED que era autoadministrado. Además, los médicos de AP recogieron datos demográficos, así como la historia clínica incluyendo información relativa a posibles factores de riesgo relacionados con la DE (enfermedades concomitantes, factores de riesgo cardiovascular, medicación concomitante, etc.). El paciente era remitido por el médico de AP a una visita con el urólogo, entre 1 y 4 semanas después, en la cual éste constataba o no el diagnóstico a partir de una entrevista clínica en profundidad. Los pacientes debían completar de nuevo el cuestionario SQUED y además el cuestionario IIEF.

El estudio de validación psicométrica del cuestionario incluyó la participación de 13 urólogos y 92 médicos de AP españoles. El estudio fue aprobado por el Comité Ético del Hospital Carlos Haya, los Comités de Ética correspondientes a todos los hospitales participantes fueron informados, y se llevó a cabo de acuerdo con las directrices internacionales de estudios epidemiológicos. Todos los sujetos firmaron el consentimiento informado.

\section{Cuestionario SQUED}

El cuestionario SQUED consta de 3 preguntas referidas a los últimos 6 meses (Tabla 1) con respuestas que oscilaban entre 'Ni lo intentó' $(0$ puntos) hasta 'Casi siempre o siempre' (5 puntos). La puntuación de corte para clasificar a un sujeto como DE fue inicialmente establecida en 13 puntos, considerando que los sujetos con puntuaciones superiores podía considerarse que no estaban afectados de DE.

El desarrollo del cuestionario SQUED se realizó como sigue:

1. Identificación de conceptos y generación de items (versión 1). Después de una revisión exhaustiva de la documentación existente sobre DE, se llevó a cabo una reunión con un urólogo con experiencia clínica en DE para:

- Elaborar la lista de conceptos o dominios relevantes para identificar la presencia o ausencia de DE.

- Decidir el orden en que dichos conceptos debían preguntarse.

- Formato de respuestas.

- Referencia temporal. 
Tabla 1

Cuestionario SQUED

\begin{tabular}{|c|c|c|c|c|c|}
\hline \multicolumn{6}{|c|}{ Durante los últimos 6 meses, ¿con qué frecuencia le ha sido posible la penetración? } \\
\hline Ni lo intentó & Nunca & $\begin{array}{l}\text { Menos de la } \\
\text { mitad de las } \\
\text { veces }\end{array}$ & $\begin{array}{c}\text { Aproximadamente } \\
\text { la mitad de las } \\
\text { veces }\end{array}$ & $\begin{array}{l}\text { Más de la mitad } \\
\text { de las veces }\end{array}$ & $\begin{array}{c}\text { Casi siempre } \\
\text { o siempre }\end{array}$ \\
\hline 0 & 1 & 2 & 3 & 4 & 5 \\
\hline
\end{tabular}

Durante los últimos 6 meses, ¿con qué frecuencia le ha sido posible mantener la erección hasta finalizar el acto sexual?

\begin{tabular}{|c|c|c|c|c|c|}
\hline Ni lo intentó & Nunca & $\begin{array}{l}\text { Menos de la } \\
\text { mitad de las } \\
\text { veces }\end{array}$ & $\begin{array}{c}\text { Aproximadamente } \\
\text { la mitad de las } \\
\text { veces }\end{array}$ & $\begin{array}{l}\text { Más de la mitad } \\
\text { de las veces }\end{array}$ & $\begin{array}{c}\text { Casi siempre } \\
\text { o siempre }\end{array}$ \\
\hline 0 & 1 & 2 & 3 & 4 & 5 \\
\hline \multicolumn{6}{|c|}{ Cuando intentó el acto sexual, ¿con qué frecuencia fue satisfactorio para usted? } \\
\hline Ni lo intentó & Nunca & $\begin{array}{l}\text { Menos de la } \\
\text { mitad de las } \\
\text { veces }\end{array}$ & $\begin{array}{c}\text { Aproximadamente } \\
\text { la mitad de las } \\
\text { veces }\end{array}$ & $\begin{array}{l}\text { Más de la mitad } \\
\text { de las veces }\end{array}$ & $\begin{array}{l}\text { Casi siempre } \\
\text { o siempre }\end{array}$ \\
\hline 0 & 1 & 2 & 3 & 4 & 5 \\
\hline
\end{tabular}

2. Control de calidad y viabilidad del cuestionario en AP. La evaluación de la viabilidad de aplicación y la opinión sobre el cuestionario fue llevada a cabo de forma independiente por tres médicos de AP. A partir de este control de calidad $\mathrm{y}$ viabilidad, algunos aspectos fueron modificados produciendo la versión 2 del cuestionario.

3. Evaluación del contenido y la validez aparente. La versión 2 del cuestionario fue evaluada de forma independiente por dos urólogos pertenecientes al comité de expertos para verificar que el contenido del cuestionario seguía contemplando todos aquellos aspectos relacionados con el concepto en estudio. Una vez incluidos los cambios propuestos (versión 3), se convocó una reunión con todo el comité de expertos involucrado en el estudio para discutir la validez aparente y de contenido de cada pregunta del cuestionario, la escala de respuesta y el referente temporal, produciendo la versión 4 .

4. Entrevistas con sujetos. Cinco sujetos con DE fueron invitados a contestar la versión 4 del cuestionario de acuerdo con la forma de aplicación prevista. Tras completar el cuestionario se realizó una entrevista en profundidad en la que se interrogaba respecto a la extensión del cuestionario y valoración global. Asimismo, para cada uno de los ítems se evaluó:

- Claridad y comprensibilidad.

- Relevancia de la pregunta.

- Ausencia de ambigüedad.
- Ausencia de términos excesivamente técnicos. - Ausencia de términos valorativos o peyorativos.

5. Elaboración de la versión final del cuestionario.

\section{Análisis estadístico}

El análisis estadístico se realizó en la población por intención de tratar (ITT), con el software SAS (versión 8).

La fiabilidad del cuestionario se valoró mediante el alfa de Cronbach que evalúa la consistencia interna, es decir, el grado en que las respuestas obtenidas con cada uno de los ítems se correlaciona con el resto del cuestionario.

La estabilidad temporal se evaluó por el método test-retest, y se calculó el índice Kappa con las dos aplicaciones del cuestionario.

La valoración de la validez del cuestionario como herramienta de detección de la DE se realizó calculando la sensibilidad del cuestionario (capacidad para clasificar correctamente los sujetos con DE) y la especificidad (capacidad para clasificar correctamente los sujetos sin DE) en relación con el diagnóstico del urólogo realizado a partir de entrevista clínica en profundidad.

Se calculó una curva ROC (Receiver's Operating Characteristics) para encontrar el punto de corte óptimo. El área bajo la curva mide la discriminación, es decir, la capacidad del cuestionario para clasificar correctamente a los sujetos con y sin la enfermedad. 


\section{RESULTADOS}

\section{Descripción de la muestra}

Fueron reclutados 405 sujetos en el estudio siendo evaluables 316 (208 con DE, 108 sin DE). No se encontraron diferencias en las características basales entre sujetos con DE y sin DE (Tabla 2), a excepción del rango de edad entre 20 y 76 (media \pm SD: $57 \pm 10$ ) y entre 19 y 80 años (media \pm SD: $53 \pm 11$ ) para sujetos con DE y sin DE, respectivamente $(\mathrm{p}=0,0002$, test de la U de MannWhitney).

La Figura 1 muestra la prevalencia de enfermedades o factores de riesgo existente en la población estudiada. La única diferencia significativa encontrada fue una mayor proporción de diabéticos entre los sujetos con DE ( $22 \%$ vs $11 \%$, $\mathrm{p}=0,016$, test de la Chi-cuadrado).

\section{Validación psicométrica}

Análisis de fiabilidad. Los 3 ítems del cuestionario de screening mostraron una alta consistencia interna (alfa de Cronbach=0,92).

La valoración de la fiabilidad test-retest mediante el análisis de puntuaciones apareadas no fue estadísticamente significativo $(\mathrm{p}=0,239$, test de la $\mathrm{t}$ de Student para datos apareados) indicando que no había cambios significativos y asegurando la estabilidad temporal del cuestionario. El índice Kappa mostró una puntuación de 0,77 , indicando buena concordancia entre el cuestionario cumplimentado en la consulta del médico de AP y el mismo cuestionario cumplimentado en la consulta del urólogo, reforzando la estabilidad temporal del cuestionario SQUED.

Tabla 2

Características basales de los sujetos

\begin{tabular}{lccc}
\hline & Edad (años) & Peso (kg) & Estatura (cm) \\
\hline Disfunción & $57,3 \pm 10,0(\mathrm{n}=212)$ & $82,5 \pm 12,8(\mathrm{n}=201)$ & $169,1 \pm 7,9(\mathrm{n}=200)$ \\
Eréctil & $58(20-76)$ & $81(58-135)$ & $170(102-185)$ \\
& & & \\
Sin Disfunción & $52,9 \pm 11,5(\mathrm{n}=108)$ & $81,0 \pm 13,6(\mathrm{n}=104)$ & $170,1 \pm 6,5(\mathrm{n}=104)$ \\
Eréctil & $53(19-80)$ & $79(50,5-130)$ & $170(155-185)$ \\
& & & \\
Global & $55,8 \pm 10,7(n=320)$ & $82,0 \pm 13,1(n=305)$ & $169,5 \pm 7-4(n=304)$ \\
& $56(19-80)^{*}$ & $80(50,5-135)$ & $170(102-185)$ \\
\hline
\end{tabular}

( $\mathrm{p}=0,0002$, test $\mathrm{U}$ de Mann-Whitney)

Media \pm Desviación estándar (número de sujetos)

Mediana (Mínimo - Máximo)

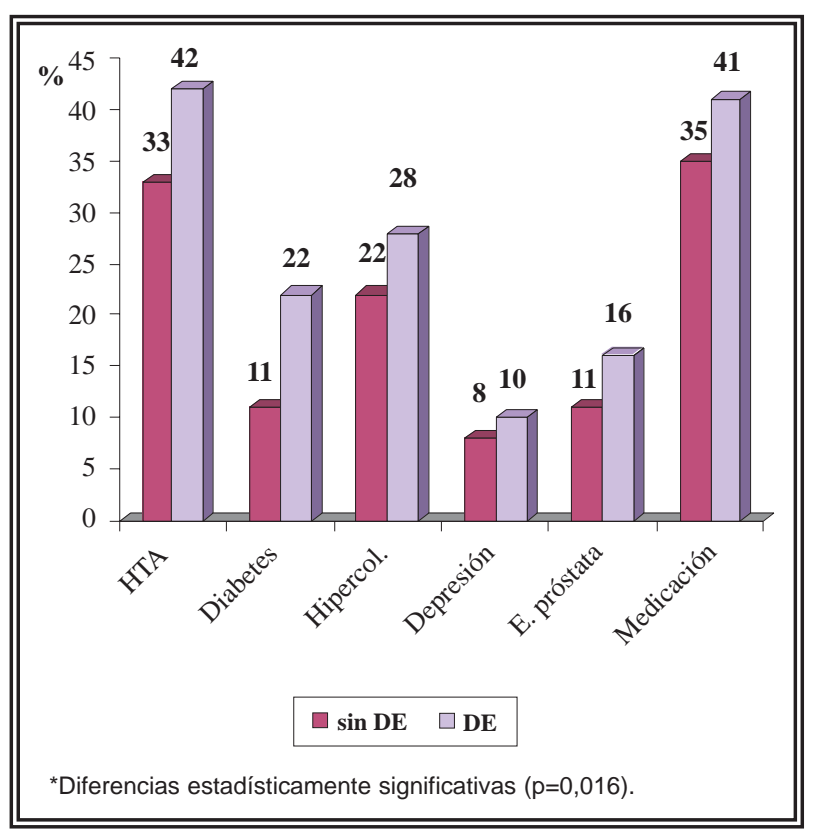

FIGURA 1. Enfermedades o factores de riesgo.

Análisis de sensibilidad y especificidad. Se obtuvieron valores altos de sensibilidad y especificidad, 0,87 y 0,78, respectivamente. Asimismo se obtuvo un elevado valor predictivo positivo (VPP $=0,88$ ), es decir, la probabilidad de que un sujeto con un resultado positivo sufra $\mathrm{DE}$, y un elevado valor predictivo negativo (VPN=0,76), probabilidad que un sujeto con un resultado negativo no tenga $\mathrm{DE}$.

Por último, el cociente de probabilidad de una prueba positiva (CPP) fue de 3,92 , indicando que en el grupo con $\mathrm{DE}$, la probabilidad de encontrar un resultado positivo es aproximadamente 4 veces superior que en el grupo sin DE. El cociente de probabilidad de una prueba negativa (CPN) fue 0,17 . Este cociente significa que un resultado negativo se encontró aproximadamente 6 veces $(1 / 0,17=5,9)$ más frecuentemente en los sujetos sin $\mathrm{DE}$ que en los sujetos con DE. Los cocientes de probabilidad relacionan sensibilidad y especificidad en un único índice que no varía con la prevalencia (Tabla 3).

En la curva ROC, el área bajo la curva obtenido en la población evaluada fue de 0,90 (Fig. 2). 
Tabla 3

Clasificación de sujetos (número de sujetos)

\begin{tabular}{lccr}
\hline $\begin{array}{c}\text { Según SQUED en } \\
\text { Atención Primaria }\end{array}$ & \multicolumn{2}{c}{ Criterio del Urólogo } & Total \\
Disfunción Eréctil & Sin Disfunción Eréctil & \\
\hline Disfunción Eréctil & 181 & 24 & 205 \\
Sin Disfunción Eréctil & 27 & 84 & 111 \\
Total & 208 & 108 & 316 \\
\hline
\end{tabular}

Function) ${ }^{9}$. Sin embargo, el cuestionario IIEF consta de 15 preguntas, seis de ellas muestran una correcta discriminación entre hombres con y sin DE, pero la capacidad discriminatoria resulta pobre para las restantes 9 preguntas y requiere más de 10 minutos para ser completado por

Esto significa que en el 90\% de los casos, un sujeto elegido al azar en el grupo con DE tendrá una puntuación superior que los sujetos sin DE (en un cuestionario perfecto con una sensibilidad y especificidad de 1, el área bajo la curva sería 1).

Examinando los diferentes puntos de corte del cuestionario, la puntuación 12 mostró el mejor equilibrio entre sensibilidad y especificidad $(0,80$ y 0,88 ), con una especificidad superior al punto de corte inicialmente seleccionado (13), aunque con una sensibilidad ligeramente inferior.

Para valorar la validez convergente, se analizó la correlación del cuestionario con aquellos instrumentos que se consideraron relacionados. La puntuación del cuestionario de detección se correlacionó significativamente con el dominio de la función eréctil del cuestionario IIEF ( $r=0,69$, p $<0,001)$.

\section{DISCUSIóN}

El cuestionario por excelencia para el diagnóstico y la valoración de la DE ha sido tradicionalmente el IIEF (International Index of Erectile

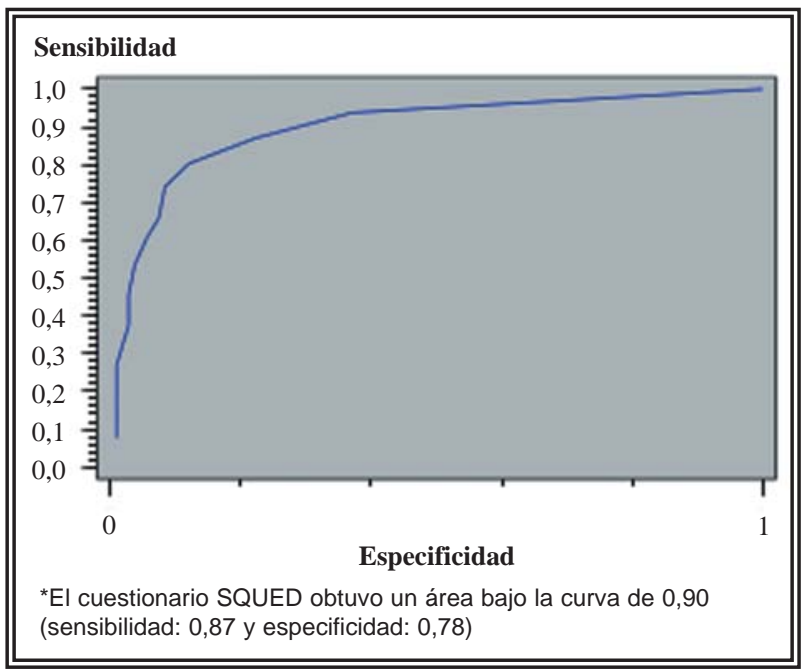

FIGURA 2. CUIVA ROC. el paciente. Posteriormente, se desarrolló una versión reducida del mismo, el IIEF- $5^{8}$, con sólo 5 preguntas, y más fácil de completar, pero con un horizonte temporal más amplio, 6 meses, en comparación con las 4 semanas del cuestionario IIEF original. La formulación de los ítems en el IIEF-5 utiliza los mismos términos que la versión original del IIEF, por lo que este cuestionario reducido presenta la misma complejidad lingüística que el inicial.

Por todo ello, disponer de una herramienta de detección de la DE aún más sencilla y comprensible y que fuera útil para el contexto de la AP, donde el tiempo por visita es muy reducido, parece necesario.

El cuestionario SQUED, con sólo 3 items que recogen los conceptos fundamentales de la definición de DE (capacidad de conseguir la erección, capacidad de mantenerla y satisfacción con la relación sexual) ha demostrado una elevada consistencia interna, estabilidad en el tiempo y una alta eficacia diagnóstica. El uso de un apropiado criterio "gold standard" basado en la historia clínica y en un examen clínico completo llevado a cabo por el médico de AP y el urólogo, además de la administración del IIEF, proporciona robustez a los resultados de sensibilidad y especificidad obtenidos para el cuestionario SQUED.

Además, se obtiene una elevada correlación positiva entre el cuestionario SQUED y el IIEF, lo que confirma que ambos cuestionarios están midiendo el mismo concepto aunque de forma mucho más rápida y sencilla en el caso del SQUED.

De los resultados obtenidos puede concluirse que el nuevo cuestionario SQUED, cumple con los objetivos de simplicidad, fiabilidad y validez diagnóstica, y puede llegar a ser un instrumento útil, y de hecho, el más sucinto validado, para la identificación de pacientes con DE tanto en el ámbito de la AP como en otros no especializados. 
Se puede esperar que el vocabulario sencillo y coloquial del cuestionario SQUED permita una fácil traducción, adaptación cultural y validación en otros idiomas, convirtiéndolo en una potencial herramienta de "detección/screening" universal, una vez los resultados psicométricos sean replicados en otros países.

\section{Agradecimientos}

A los investigadores del proyecto ESTIMA.

Urólogos: Juan José Lobato Encinas (H. General Alicante. Alicante), Eduardo Ruiz Castañé (Fundación Puigvert. Barcelona), Joan Prats López (H. Parc Taulí de Sabadell. Barcelona), Adrián de la Fuente (H. Clínico de Granada. Granada), Juan Pablo Sanz Jaca (H. Comarcal de Bidasoa. Hondarribia), Jesús Isidoro García González (H. de Móstoles. Madrid), Luis Fiter Gómez (H. Severo Ochoa de Leganés. Madrid), Antonio Martín Morales (H. Carlos Haya. Málaga), Javier Regadera Seijas (H. Central de Asturias. Oviedo), Esteban Blanco Palenciano (H. V. De la Macarena. Sevilla), Francisco Ramada Benlloch (H. General de Valencia. Valencia), José Ramón Cortiñas González (H. Clínico Universitario de Valladolid. Valladolid), Fernando Meijide Rico (Clínica Povisa. Vigo).

Médicos de Atención Primaria: Remedios Alarcón Barbero (C.S Florida. Alicante), José Rafael Berná Salas (C.S Los Angeles. Alicante), Carlos Ojea Arrendó y Francisco Llorca Catarla (C.S San Vicente. Alicante), Julio González Algas y Miguel Angel Fuentes Pérez (C.A.P Maragall. Barcelona), Carmen Berbel Bolivar (C.S Almuñecar. Granada), Manuel Alonso Ródenas (C.S Casería de Montijo. Granada), Carlos Sanchez Tovar (C.S Deifontes. Granada), José Antonio López Sánchez (C.S Loja. Granada), Antonio Pineda García (C.S Macarena. Granada), Juan Tomas Gilabert (C.S Mirasierra. Granada), José Luis Martín Manzano (C.S Salvador Caballero. Granada), Carmen Gonzalez García (C.S Tiena. Granada), José María Etxebeste Inzagaray, Fco Javier García Chivite, Antonio García Rodríguez, Agurne Linacisoro Ibarra, Enrique PérezMosso Neninger, Luis Unzueta Salvador y Arantxa Calvo Elizazu (C.S Dumboa. Irán), Jose Pérez Sanchez (C.S Loranca. Leganés), Javier Rodriguez Peso (C.S Pedroches. Leganés), Jesús Luengo Agüero (Clínica Belén. Leganés), Melitón Blasco Oliete (EAP Humanes. Leganés), Julio Sanchez Salvador (EAP $\mathrm{M}^{\mathrm{a}}$ Angeles López. Leganés), Tomas Rodelgo Cambronero (EAP Pedroches. Leganés), Fco Javier Huerta Dante (M.Jesus Hereza. Leganés), Luis Miguel de la Morena González (C.S Arroyo de la Miel. Málaga), Alvaro Milán Fernándezy Francisco Tabeada González (C.S Ciudad
Jardín-29014. Málaga), Vicente Izco López (C.S Limonar. Málaga), Pedro García Fernández y Diego Lara Navarro (C.S Nueva Málaga. Málaga), Ignacio Martínez Mérida (C.S Puerta Blanca. Málaga), Enrique Martos Gutiérrez (C.S San Miguel. Málaga), Inmaculada Sanchez Hernández (C.S Valle de Abdalagis. Málaga), Josefina Aparicio Velasco, Jose Manuel Méntrida Rodríguez y M $\mathrm{M}^{\mathrm{a}}$ Carmen Marin Osorio (C.S Alcalde Bartolomé. Móstoles), Juan Carlos Muñoz García (C.S Azorin. Móstoles), Francisco Rodrigo Jiménez y Alfredo Falcó Sales (C.S Barcelona. Móstoles), Daniel Serrano Tomas, Gregorio Perdices Pinilla y Pablo Romero Martínez (C.S Dos de Mayo. Móstoles), Gonzalo Zarate Valenzuela (C.S Presentación Sabio. Móstoles), Ramón Alonso Gómez (C.S Noreña. Oviedo), Ignacio González Escandón (C.S Posada de Llanera. Oviedo), Jose Antonio Fernández Fernández (C.S Pumarin. Oviedo), Eduardo Murias Siñeriz (C.S Sta. Eulalia. Oviedo), Joaquín Cuetos Alvarez (Consultorio de Valdesoto. Oviedo), Miguel Angel Fernández Fernández (Jefatura Superior. Oviedo), Javier Ignacio Garrido Peon (Sanidad Guardia Civil. Oviedo), Maria Jesús Cubells Larrosa (ABS Lepant. Sabadell), Francesc Solé Llorens (C.S Privada. Sabadell), Antonio Moya Moya y Antonio Salido Cano (CAP Castellar del Vallès. Sabadell), Miguel González Herrero y Esperanza Fernández Barrio (CAP Concordia. Sabadell), Joseph Palou Torrentó (CAP Rambla Sabadell. Sabadell), Rufino Orús Loncá (ICS Mutuam. Sabadell), Jose Manuel Toro Barragán y Enrique Diaz Monge (C.S Coria del Río. Sevilla), Manuel Cancio Luque (C.S Dr. Fleming. Sevilla), Adán González Huambos (C.S El Porvenir. Sevilla), Blas Poyatos Poyatos (C.S Polígono Norte. Sevilla), Juan Manuel Miras Ruiz y Jose Joaquín Masa Sanchez (C.S San José Rinconada. Sevilla), Antonio Lemus Gallego (C.S San Pablo. Sevilla), Carlos Almendro Padilla, Joaquin Casado Gonzalez, José Isidoro Martínez Olivares, Miguel Angel Furió Soriano, Fernando Roselló Roca, $M^{\mathbf{a}}$ Dolores Alfonso Doménech y Concha Estellés Dasí (C.S Torrent. Valencia), M ${ }^{\mathbf{a}}$ Teresa García Gonzalo y Amaya Narbaiza Garzabal (C.S Circular. Valladolid), Ricardo García Alonso (C.S de Olmedo. Valladolid), $\mathrm{M}^{\mathbf{a}}$ Concepción Gil San Martín (C.S Delicias I. Valladolid), Pedro García de Padilla (C.S Pilanza. Valladolid), Jose Carlos Minguez Villaiz (C.S Tortola. Valladolid), Manuel González-Alegre Díez, Gerardo Miniño Couto y Enrique Cobas Varela (C.S Beiramar. Vigo), Federico Cardelle García (C.S Casa de Mar. Vigo), Lino Fontaína Pérez (C.S Coia. Vigo), Carmen Pache Muínos y Rosa Ledo Rivas (C.S Coruxo. Vigo), Manuel Bayón Meleiro (C.S López Mora. Vigo), Sagrario Franco Malvar (C.S Val de Miñor. Vigo). 


\section{REFERENCIAS}

1. Impotence. NIH Consens Statement. 1992;7-9;10(4):1-34.

2. Feldman HA, Goldstein I, Hatzichristou DG, Krane RJ, McKinlay JB. Impotence and its medical and psychosocial correlates: results of the Massachusetts Male Aging Study. J Urol. 1994;151(1):54-61.

3. Ponholzer A, Temml C, Mock K, Marszalek M, Obermayr R, Madersbacher S. Prevalence and risk factors for erectile dysfunction in 2869 men using a validated questionnaire. Eur Urol. 2005;47(1):80-86.

4. Martin-Morales A, Sanchez-Cruz JJ, Saenz de Tejada I, Rodriguez-Vela L, Jimenez-Cruz JF, Burgos-Rodriguez R. Prevalence and independent risk factors for erectile dysfunction in Spain: results of the epidemiology of erectile dysfunction. J Urol. 2001;166(2):569-74; discussion 574575.

5. Gil A, Martinez E, Oyagüez I, Palacios G, Rejas J. Erectile dysfunction in a primary care setting: results of an observational, no-control-group, prospective study with sildenafil under routine conditions of use. Int J Impot Res. 2001; 13(6):338-347.

6. Klotz T, Sachse R, Heidrich A, Jockenhövel F, Rohde G, Wensing $\mathrm{R}$, et al. Vardenafil increases penile rigidity and tumescence in erectile dysfunction patients: a RigiScan and pharmacokinetic study. World J Urol. 2001;19(1):32-39.

7. Bischoff E, Niewoehner U, Haning H, Es Sayed M, Schenke T, Schlemmer KH. The oral efficacy of Vardenafil hydrochloride for inducing penile erection in a conscious rabbit model. J Urol. 2001;165(4):1316-1318.

8. Rosen RC, Cappelleri JC, Smith MD, Lipsky J, Pena BM. Development and evaluation of an abridged, 5-item version of the International Index of Erectile Function (IIEF-5) as a diagnostic tool for erectile dysfunction. Int $J$ Impot Res. 1999;11(6):319-326.

9. Rosen RC, Riley A, Wagner G, Osterloh IH, Kirkpatrick J, Mishra A. The international index of erectile function (IIEF): a multidimensional scale for assessment of erectile dysfunction. Urology. 1997;49(6):822-830.

Correspondencia autor: Dra. M. Artés Ferragud. Adelphi Targis. C/Aragón, 182, $7^{\text {a }}$ planta, 08011. Barcelona. Tel.: 934523911 Fax: 934516304

E-mail autor: maite.artes@adelphi-targis.com Información artículo: Original - Disfunción eréctil Trabajo recibido: noviembre 2006 Trabajo aceptado: diciembre 2006 